\title{
Temperature stabilization requirements for unchopped thermal detectors
}

\author{
Marc C. Foote" \\ Center for Space Microelectronics Technology, Jet Propulsion Laboratory \\ California Institute of Technology, Pasadena, CA 91109-8099
}

\begin{abstract}
The temperature stabilization requirements of unchopped thermistor bolometers and thermopile detectors are analyzed. The detector temperature, on which the bolometer output signal depends, is quite sensitive to changes in instrument temperature but relatively insensitive to changes in scene temperature. In contrast, the difference in temperature between detector and substrate (instrument), on which the thermopile signal depends, is equally sensitive to changes in instrument and scene temperature. Expressions for these dependencies are derived based on a simplified instrument model. It is shown that for a typical uncooled thermal imager, the temperature stabilization requirements for a bolometer are two orders of magnitude more stringent than those for a thermopile detector.
\end{abstract}

Keywords: thermistor, bolometer, thermopile, detector, IR, thermal, temperature stabilization

\section{INTRODUCTION}

The thermal detector class includes thermistor bolometers, pyroelectric and ferroelectric detectors, and thermopiles. Each has a thermally isolated absorbing structure that heats up upon absorption of incident radiation. This temperature change is sensed by one of several methods. A thermistor bolometer, hereafter referred to as a bolometer, senses the temperature of the absorber with a temperature dependent resistive material. A pyroelectric or ferroelectric detector produces a voltage signal dependent on the rate of absorber temperature change. A thermopile measures yet another property - the difference in temperature between the thermally isolated absorber and a reference heat sink (usually the detector substrate). Because the three types of detectors measure different properties related to the absorber temperature, the implementation of each type of detector is different. The fact that pyroelectric detectors respond only to time-varying signals necessitates chopped incident radiation. Often a chopper is undesirable. In this analysis we focus on unchopped systems utilizing bolometers or thermopile detectors. It is often stated that bolometers require temperature stabilization while thermopiles do not. The purpose of this paper is to quantify the temperature stabilization requirements for the two types of detectors in order to aid in detector selection and application.

The current detector of choice for uncooled imaging applications is the Honeywell-developed ' bolometer array. One reason for this choice is the relative simplicity of readout circuitry for a $2 \mathrm{D}$ bolometer array compared to a thermopile array. A bolometer requires a current (or voltage) bias and the responsivity is proportional to this bias. Instead of applying a constant current bias, a bolometer array can be read out sequentially by applying a large current bias to each pixel for a short period of time. The current amplitude and pulse duration are such that the average power dissipated at the pixel is the same as the constant bias case. The increase in signal due to the larger bias approximately equals the increase in noise due to the larger electrical bandwidth. Thus, bolometer arrays can be read out sequentially without significant degradation in signal-to-noise ratio. A thermopile, while not requiring a bias, has no such way to increase its response. Therefore, to achieve a high signalto-noise ratio, each pixel must have a dedicated low-noise amplifier, with multiplexing after this initial electronics stage.

A second advantage of bolometers over thermopiles is sensing materials. The vanadium oxide material used in bolometer arrays has good performance and is compatible with semiconductor fabrication processes. Most existing thermopile arrays use silicon based ${ }^{2-6}$ or metal ${ }^{7.9}$ thermoelectric materials. While these materials are also compatible with silicon processes, they offer only moderate detector performance. The thermopile arrays with highest performance incorporate bismuth-based thermoelectric materials, ${ }^{9.10}$ which may be more difficult to combine with silicon processes.

A disadvantage of bolometers is that they require tight temperature stabilization. This requirement increases the complexity and power of a bolometric system. Since a major thrust of the uncooled imaging effort is to produce compact, low cost

\footnotetext{
Correspondence: Email: marc.c.foote @jpl.nasa.gov; Telephone: (818) 354-9009
} 
systems, temperature stabilization will eventually become an important issue. Some reduction in bolometer stabilization requirements can be obtained with reference bolometers on the substrate and with software corrections for temperature drifts. However, the use of thermopiles dramatically reduces the need for this effort. Other advantageous features of thermopiles are the generation of signal without electrical bias, lack of output voltage pedestal, lack of $1 / \mathrm{f}$ noise, and high linearity.

In the remainder of this paper a model instrument employing thermal detectors is analyzed. Based on this model the detector temperature (temperature of the thermally isolated absorber), $T_{D}$, is calculated. It is shown that the detector temperature is determined predominantly by the instrument temperature, and only slightly by the scene temperature. Since bolometers measure $T_{D}$, the conclusion is that the bolometer signal is much more sensitive to changes in the instrument temperature than it is to changes in the scene temperature. Hence careful temperature stabilization is required. In contrast, the difference in temperature between the detector and instrument (substrate), $\Delta T$, is equally sensitive to changes in the instrument or scene temperature. A thermopile signal, proportional to $\Delta T$, is therefore influenced by the instrument and scene temperature equally. Thus, correction for instrument temperature drifts in thermopile instruments is fairly straightforward. It is shown for a typical uncooled thermal imager that the temperature stabilization or correction requirements for a bolometer are two orders of magnitude more stringent than those required for a thermopile detector.

\section{INSTRUMENT MODEL}

Figure 1 shows an idealized infrared instrument containing a single thermal detector, which could be a thermopile, a thermistor bolometer, or a pyroelectric (ferroelectric) detector. This thermally isolated detector, with area $A$ and temperature $T_{D}$, has a front-side emissivity $\varepsilon_{D}$ and back-side emissivity $\varepsilon_{B}$. The detector is connected to the instrument through a physical support with thermal conductance $G_{k}$, assumed to be temperature independent. This support is typically two narrow silicon nitride legs connecting a thermally isolated membrane detector to the substrate. For simplicity it is assumed that the substrate and instrument housing are at a uniform temperature $T_{1}$ and have emissivity $\varepsilon_{l}$. The detector is radiatively coupled to the scene, which has temperature $T_{s c}$ and emissivity $\varepsilon_{S_{c}}$, through an optic with $\mathrm{f} / \#=f$. The optic has transmission $\tau$ and reflectivity $r$ such that $\tau+r=1$. Outside the optic f-cone the detector sees only the instrument housing. The radiative coupling between detector and scene changes the detector temperature with respect to the instrument. If the scene is warmer than the instrument then the difference between detector and instrument temperature, $T_{D}-T_{1}=\Delta T$, is positive. A cold scene will produce a negative value of $\Delta T$.

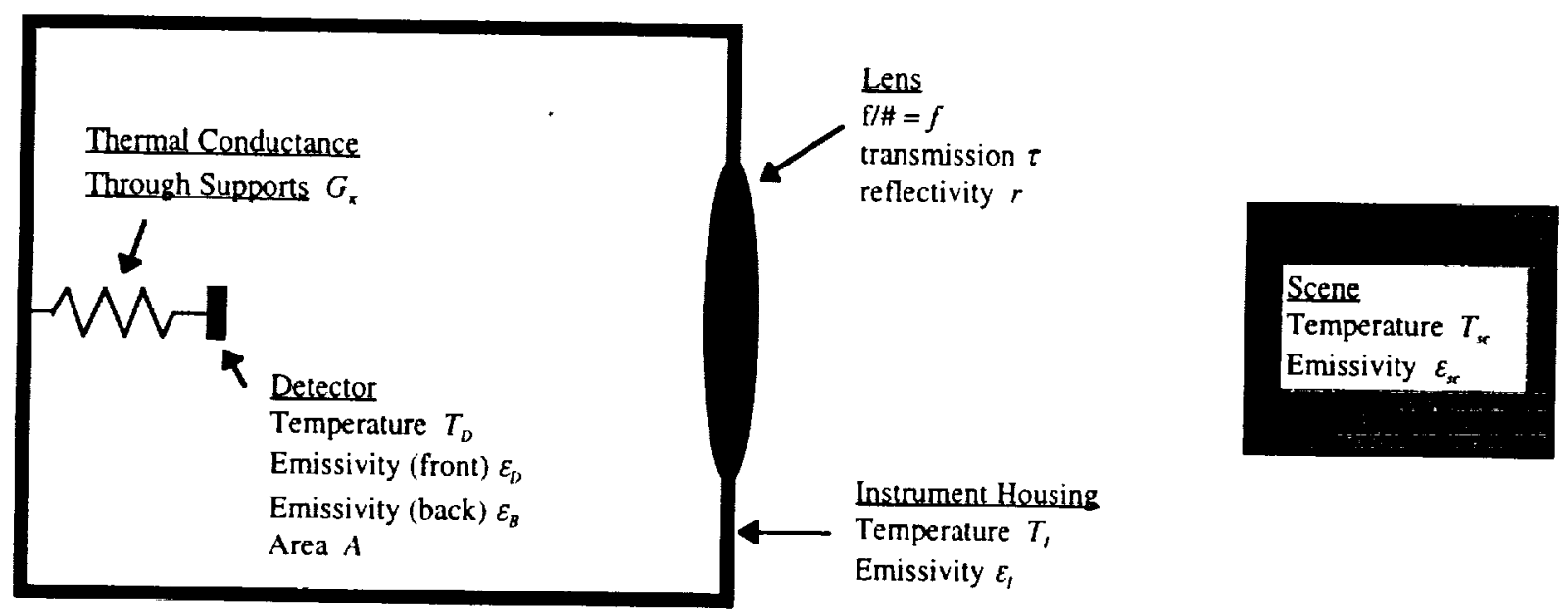

Figure I. Simplified model used in calculations. 


\section{OUTPUT SIGNAL FROM THERMOPILES AND BOLOMETERS}

The voltage output from a current biased, unchopped bolometer is

$$
V_{B o l}=I \mathrm{R}_{1} T_{o}\left[1+\alpha\left(T_{D}-T_{o}\right)\right]=I \mathrm{R}_{\left(T_{o}\right)}\left(1-\alpha T_{o}\right)+I \alpha \mathrm{R}_{\left(T_{o}\right)} T_{D}
$$

where I is the bias current, $R_{(}\left(T_{o}\right)$ is the thermistor resistance at a reference temperature $T_{a}$ near $T_{D}$, and $\alpha$ is the temperature coefficient of resistance of the thermistor element. This bolometer output voltage has a constant offset term plus a term proportional to the detector temperature $T_{D}$.

A thermopile detector has $N$ thermocouples connected in series, each running from the substrate to the thermally isolated absorber. The output voltage signal is given by

$$
V_{\text {therm }}=N S\left(T_{D}-T_{l}\right)=N S \Delta T
$$

where $S$ is the Seebeck coefficient for a single thermocouple, expressed as voltage generated across the thermocouple per degree $K$ of temperature difference between hot and cold junctions.

For an unchopped system, then, a bolometer output depends on $T_{D}$ while a thermopile output depends on $\Delta T$. The following analysis shows that devices depending on $\Delta T$ are much less sensitive to instrument temperature changes than devices depending on $T_{D}$. Note that if optical chopping is used with either a bolometer or pyroelectric detector, the amplitude of the output ac signal is proportional to $\Delta T$. The advantage of the thermopile is that chopping is not required.

\section{DERIVATION OF EXPRESSIONS FOR $T_{b}$ AND $\Delta T$}

To determine the detector temperature, consider the balance of heat flowing into and out of the detector. Positive power indicates heat flowing into the detector; negative power indicates heat flowing out of the detector. Heat power flowing
through the detector supports is

$$
P_{G}=-\Delta T G_{\kappa} \text {. }
$$

Power radiated from the detector is given by

$$
P_{\text {Rad }}=-\left[\varepsilon_{D}+\varepsilon_{B}\right] A \sigma T_{D}^{4}
$$

where $\sigma$ is the Stefan-Boltzmann constant. Radiative power absorbed by the detector is

$$
\dot{P}_{A b s}=\varepsilon_{l} \varepsilon_{B} A \sigma T_{l}^{4}+\frac{\varepsilon_{S_{c}} \varepsilon_{D} \tau A \sigma T_{S_{c}}^{4}}{4 f^{2}+1}+\frac{\varepsilon_{l} \varepsilon_{D} r A \sigma T_{I}^{4}}{4 f^{2}+1}+\frac{4 f^{2} \varepsilon_{l} \varepsilon_{D} A \sigma T_{l}^{4}}{4 f^{2}+1} .
$$

In this expression the first term represents power radiated by the instrument housing and absorbed by the back side of the detector. The second term represents radiation from the scene transmitted through the lens and absorbed by the detector. The third term also represents radiation within the lens $f$ cone, but is instrument radiation reflected from the lens. The fourth term is radiation from the instrument, incident outside the lens $f$ cone. At steady state the sum of all powers to and from the
detector is zero.

$$
P_{G}+P_{R a d}+P_{A b s}=0
$$
After a sudden change in the scene temperature, this steady state will be reached with a $1 / e$ time equal to the detector
response time.

We will assume for simplicity that $\varepsilon_{t}=1$. Substituting Equations $3-5$ into Equation 6 and rearranging gives

$$
\Delta T G_{\kappa}+A \sigma\left[\varepsilon_{B}+\varepsilon_{D}\left(\frac{4 f^{2}+r}{4 f^{2}+1}\right)\right]\left(T_{D}^{4}-T_{i}^{4}\right)+\frac{\varepsilon_{D} A \sigma \tau\left(T_{D}^{4}-\varepsilon_{S_{c}} T_{S_{c}}^{4}\right)}{4 f^{2}+1}=0
$$
Equation 7 can be simplified because the detector temperature deviates only slightly from the instrument (substrate)
temperature. Using the approximation

$$
T_{D}^{4} \approx T_{l}^{+}+4 T_{l}^{3} \Delta T
$$

Equation 7 becomes 


$$
\left[G_{\kappa}+4 A \sigma\left(\varepsilon_{B}+\varepsilon_{D}\right) T_{l}^{3}\right] \Delta T+\frac{\varepsilon_{D} A \sigma \tau\left[T_{l}^{4}-\varepsilon_{S c} T_{S r}^{4}\right]}{4 f^{2}+1}=0 .
$$

The total thermal conductance $\left.G_{(} T_{1}\right)$ from the detector is.

$$
G_{\left(T_{l}\right)}=G_{x}+4 A \sigma\left(\varepsilon_{B}+\varepsilon_{D}\right) T_{l}^{3},
$$

so $\Delta T$ is equal to

$$
\Delta T=-\frac{\varepsilon_{D} A \sigma \tau\left[T_{l}^{4}-\varepsilon_{S_{r}} T_{S_{r}}^{4}\right]}{G_{\left(T_{1}\right)}\left(4 f^{2}+1\right)}
$$

and the detector temperature $T_{D}$ is

$$
T_{D}=T_{l}-\frac{\varepsilon_{D} A \sigma \tau\left[T_{l}^{4}-\varepsilon_{S c} T_{S_{c}}^{4}\right]}{G_{\left(T_{l}\right)}\left(4 f^{2}+1\right)}
$$

\section{SENSITIVITY OF $T_{D}$ TO CHANGES IN SCENE AND INSTRUMENT TEMPERATURE}

Regardless of thermal detector type, the sensitivity of detector temperature $T_{D}$ to changes in the scene temperature is the derivative of Equation 12 with respect to $T_{s \boldsymbol{c}}$.

$$
\frac{\partial T_{D}}{\partial T_{S_{c}}}=\frac{4 \varepsilon_{D} \varepsilon_{S_{c}} A \sigma \tau T_{S_{c}}^{3}}{G_{\left(\tau_{l}\right)}\left(4 f^{2}+1\right)}
$$

while the sensitivity of detector temperature to changes in the instrument temperature is the derivative of Equation 12 with respect to $T_{1}$.

$$
\frac{\partial T_{D}}{\partial T_{I}}=1-\frac{4 \varepsilon_{D} A \sigma \tau T_{I}^{3}}{G_{\left(T_{I}\right)}\left(4 f^{2}+1\right)}\left[1-\frac{3\left(\varepsilon_{D}+\varepsilon_{B}\right) A \sigma\left(T_{l}^{4}-\varepsilon_{D} T_{S c}^{4}\right)}{T_{l} G_{\left(T_{l}\right)}}\right]
$$

For the detector temperature $T_{D}$ (measured directly by a bolometer), the ratio of instrument temperature sensitivity to scene temperature sensitivity is therefore given by the ratio of Equations 14 and 13 .

$$
\left.\frac{\left(\partial T_{D} / \partial T_{I}\right)}{\left(\partial T_{D} / \partial T_{S_{c}}\right)}=\left[\frac{G_{\left(T_{l}\right)}\left(4 f^{2}+1\right)}{4 \varepsilon_{D} A \sigma \tau T_{i}^{3}}-1+\frac{3\left(\varepsilon_{D}+\varepsilon_{B}\right) A \sigma\left(T_{I}^{4}-\varepsilon_{D} T_{S_{c}}^{4}\right)}{T_{l} G_{\left(T_{l}\right)}}\right] \frac{T_{I}^{3}}{\varepsilon_{S_{c}} T_{S_{c}}^{3}}\right)
$$

For illustration, consider the case of an uncooled bolometric thermal imager. Typical values are $\varepsilon_{D}=0.8, A=0.5 \times(50 \mu m)^{2}$ (= fill factor times total pixel area), $G=10^{-7} \mathrm{~W} / \mathrm{K}, T_{l}=T_{\mathrm{Sc}_{\mathrm{c}}}=300 \mathrm{~K}, \tau=0.9$, and $f=1$. Assuming $\varepsilon_{\mathrm{Sc}}=0.8$, Equation 15 is equal to

$$
\frac{\left(\partial T_{D} / \partial T_{l}\right)}{\left(\partial T_{D} / \partial T_{S_{c}}\right)}=113
$$

Thus, for a typical uncooled bolometric thermal imager, the detector signal is about two orders of magnitude more sensitive to changes in instrument temperature than to changes in scene temperature. If, for example, a temporal noise-equivalent temperature difference (NETD) of $50 \mathrm{mK}$ is required when looking at a scene with $\varepsilon_{\mathrm{sc}_{\mathrm{c}}}=1$, then the instrument temperature must be stable (or correctable) to $50 \mathrm{mK} / 91=0.55 \mathrm{mK}$. Note that an optics transmission of 0.9 is actually an optimistic assumption since the transmission decreases outside the 8-12 $\mu \mathrm{m}$ region. Lower transmission will make $T_{D}$ less sensitive to scene temperature and will increase the ratio in Equation 16.

Now consider the case where the scene temperature is low compared to the instrument temperature. An example of this situation is an ambient-temperature spacecraft instrument imaging a cold body. As the scene temperature decreases from that 
of the instrument, the ratio of the instrument temperature sensitivity to the scene temperature sensitivity, given in Equation 15, increases roughly as $T_{i}^{3} / T_{s i}{ }^{3}$. Therefore the required instrument temperature stabilization becomes proportionally more stringent. Similarly, for scene temperatures higher than the instrument temperature, such as is common in industrial applications, the temperature stabilization requirements relax roughly as $T_{l}^{3} / T_{s_{c}}{ }^{3}$.

\section{SENSITIVITY OF $\Delta T$ TO CHANGES IN SCENE AND INSTRUMENT TEMPERATURE} The sensitivity of temperature difference $\Delta T$ to changes in the scene temperature is the derivative of Equation 11 with respect
to $T_{S_{r}}$.

$$
\frac{\partial(\Delta T)}{\partial T_{S_{r}}}=\frac{4 \varepsilon_{D} \varepsilon_{S_{r}} A \sigma \tau T_{S_{r}}^{3}}{G_{\left(T_{1}\right)}\left(4 f^{2}+1\right)}
$$

while the sensitivity of $\Delta T$ to changes in the instrument temperature is the derivative of Equation $1 !$ with respect to $T_{1}$.

$$
\frac{\partial(\Delta T)}{\partial T_{l}}=-\frac{4 \varepsilon_{D} A \sigma \tau T_{l}^{3}}{G_{\left(T_{l}\right)}\left(4 f^{2}+1\right)}\left[1-\frac{3\left(\varepsilon_{D}+\varepsilon_{B}\right) A \sigma\left(T_{l}^{4}-\varepsilon_{S_{c}} T_{S_{c}}^{4}\right)}{T_{l} G_{\left(T_{l}\right)}}\right]
$$

For the temperature difference $\Delta T$ (measured directly by a thermopile), the ratio of instrument temperature sensitivity to scene temperature sensitivity is therefore given by the ratio of Equations 18 and 17 .

$$
\frac{\left(\partial(\Delta T) / \partial T_{l}\right)}{\left(\partial(\Delta T) / \partial T_{S_{c}}\right)}=-\frac{T_{l}^{3}}{\varepsilon_{S_{c}} T_{S_{c}}^{3}}\left[1-\frac{3\left(\varepsilon_{D}+\varepsilon_{B}\right) A \sigma\left(T_{l}^{4}-\varepsilon_{S_{c}} T_{S_{c}}^{4}\right)}{T_{l} G_{\left(T_{l}\right)}}\right]
$$

Considering again a typical uncooled thermal imager looking at a scene with emissivity 0.8 , Equation 19 is

$$
\frac{\left(\partial(\Delta T) / \partial T_{1}\right)}{\left(\partial(\Delta T) / \partial T_{S_{c}}\right)}=-1.2
$$

Thus, for a typical uncooled thermopile thermal imager, the detector signal is equally sensitive to instrument temperature and scene temperature. If, for example, a temporal NETD of $50 \mathrm{mK}$ is required when looking at a scene with $\varepsilon_{s c}=1$, then the instrument temperature must be stable (or correctable) to about $42 \mathrm{mK}$. Again, the $T_{i}^{3} / T_{\mathrm{sc}}{ }^{3}$ term results in more temperature stabilization required for low scene temperatures, and less required for high scene temperatures.

\section{STABILIZATION REQUIREMENT COMPARISON FOR BOLOMETERS AND THERMOPILES}

The quantities $T_{D}$ and $\Delta T$ are equally sensitive to changes in scene temperature, but have different sensitivities to changes in instrument temperature. One can define a factor $F$ which represents the ratio of instrument temperature sensitivities for the two quantities. $F$ then quantifies the increased temperature stabilization (or correction) requirements of a bolometer over those for a thermopile. This factor is the absolute value of the ratio of Equations 14 and 18 .

$$
F=\frac{G_{\left(T_{1}\right)\left(4 f^{2}+1\right)}}{4 \varepsilon_{D} A \sigma \tau T_{1}^{3}\left[1-\frac{3\left(\varepsilon_{D}+\varepsilon_{B}\right) A \sigma\left(T_{I}^{4}-\varepsilon_{s c} T_{S c}^{4}\right)}{T_{l} G_{\left(T_{1}\right)}}\right]}-1
$$

Note that $\mathrm{F}$ is roughly proportional to the square of the optics f number. Consequently, as $f$ increases, the advantage of the thermopile increases. To gain more physical insight, we define a quantity $g\left(T_{1}\right)$ which is the ratio of $G\left(T_{1}\right)$ and the radiation limited value of $G\left(T_{1}\right)$.

$$
g_{\left(T_{1}\right)}=\frac{G_{\left(T_{1}\right)}}{4\left(\varepsilon_{D}+\varepsilon_{B}\right) A \sigma T_{i}^{3}}
$$
An ideal thermal detector has $g\left(T_{1}\right)=1$. A typical uncooled imager as described in Section 5 has $g\left(T_{1}\right)=16$. Equation 21
becomes 


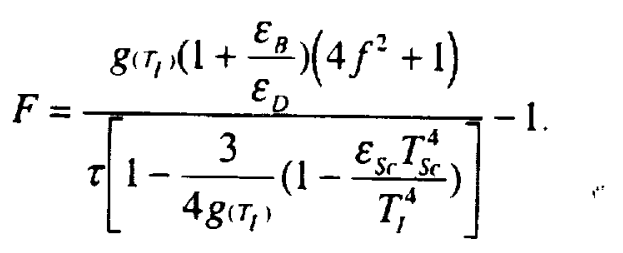

We can calculate the factor $F$ for different regimes of scene temperature. If the scene temperature is about equal to the instrument temperature, such as in an uncooled thermal imager for ambient temperature scenes, then

$$
F\left(t_{S r}=T_{l}\right)=\frac{g\left(\tau_{l}\right)\left(1+\frac{\varepsilon_{B}}{\varepsilon_{D}}\right)\left(4 f^{2}+1\right)}{\tau}-1
$$

For a typical uncooled thermal imager, with $g\left(T_{1}\right)=16, F$ is about 100 for $\mathrm{f} / 1$ optics. Hence a bolometer is about 100 times more sensitive than a thermopile to instrument temperature, so the bolometer stabilization requirements are 100 times tighter. The most favorable case for the bolometer is when the thermal paths are purely radiative and $g\left(T_{1}\right)=1$. Then $F$ is about 5 for $\mathrm{f} / 1$ optics. Thus, improved detector thermal isolation decreases the bolometer stabilization requirements.

For scene temperatures low compared to the instrument temperature,

$$
F\left(T_{S c}<T_{l}\right) \approx \frac{g_{\left(T_{l}\right)}\left(1+\frac{\varepsilon_{B}}{\varepsilon_{D}}\right)\left(4 f^{2}+1\right)}{\tau\left[1-\frac{3}{4 g\left(T_{l}\right)}\right]}-1 .
$$
A typical uncooled imager with $g\left(T_{l}\right)=16$, looking at a cold scene, has $F$ of about 100 for $f / 1$ optics. If $g\left(T_{l}\right)=1$, then $F$ is
about 20 for $f / 1$ optics.

For some industrial applications where the scene is much hotter than the instrument,

$$
F\left(T_{S c}>T_{l}\right) \approx \frac{g_{\left(T_{l}\right)}\left(1+\frac{\varepsilon_{B}}{\varepsilon_{D}}\right)\left(4 f^{2}+1\right)}{\tau\left[1+\frac{3 \varepsilon_{S_{c}} T_{S_{c}}^{4}}{4 g_{\left(T_{l}\right)} T_{l}^{4}}\right]}-1 .
$$

For an instrument temperature of $300 \mathrm{~K}$, a scene temperature of $1000 \mathrm{~K}$, and $g\left(T_{l}\right)=16$ (typical uncooled imager), $F$ is about 10 for $\mathrm{t} / 1$ optics. For larger scene temperature or smaller $g\left(T_{1}\right)$ the approximations of $\Delta T \ll\left|T_{D}-T_{1}\right|$ breaks down.

\section{SUMMARY}

We have quantified the temperature stabilization requirements for unchopped bolometers and thermopile detectors. Although bolometer readout circuitry for $2 \mathrm{D}$ arrays is simpler than that for thermopiles, bolometers require much stricter temperature control or correction. Specifically, for a typical uncooled thermal imager, the bolometer requires about two orders of magnitude stricter temperature control or correction. For such an imager with a NETD of $50 \mathrm{mK}$, the bolometer array requires temperature stability to about $0.5 \mathrm{mK}$, while a thermopile array only requires $42 \mathrm{mK}$ stability. For all thermal detectors, the ratio of instrument temperature sensitivity to scene temperature sensitivity varies roughly as $1 / T_{S i}{ }^{3}$. Thus, higher temperature scenes result in relaxed stabilization requirements while lower temperature scenes result in increased stabilization requirements. In all cases, bolometers require more stabilization than thermopiles. The difference between bolometers and thermopiles increases roughly as the square of the optics $f$ number. This difference decreases as the detector thermal isolation approaches the radiative limit. However, in the most favorable case analyzed, an unchopped bolometer still requires several times tighter temperature stabilization than a thermopile detector 


\section{ACKNOWLEDGMENTS}

The research described in this paper was performed by the Center for Space Microelectronics Technology, Jet Propulsion Laboratory, California Institute of Technology, and was sponsored by the National Aeronautics and Space Administration,
Office of Space Science.

\section{REFERENCES} 1. R.A. Wood, "Uncooled thermal imaging with monolithic silicon focal planes", Proc. SPIE 2020, Infrared Technology
XIX, pp. 322-329, 1993.

2. I.H. Choi and K.D. Wise, "A Silicon-Thermopile-Based Infrared Sensing Array for Use in Automated Manufacturing", IEEE Transactions on Electron Devices, vol. ED-33, pp. 72-79, 1986.

3. P.M. Sarro, H. Yashiro, A.W. v. Herwaarden and S. Middelhoek, "An Integrated Thermal Infrared Sensing Array", Sensors and Actuators, vol. 14, pp. 191-201, 1988.

4. T. Kanno, M. Saga, S. Matsumoto, M. Uchida, N. Tsukamoto, A. Tanaka, S. Itoh, A. Nakazato, T. Endoh, S. Tohyama, Y. Yamamoto, S. Murashima, N. Fujimoto, and N. Teranishi, "Uncooled infrared focal plane array having $128 \times 128$ thermopile detector elements", Proc. SPIE Vol. 2269, Infrared Technology XX , pp. 450-459, 1994.

5. Wayne G. Baer, Khalil Najafi, Kensall D. Wise and Robert $S$. Toth, "A 32-element micromachined thermal imager with on-chip multiplexing". Sensors and Actuators A, vol. 48, pp. 47-54, 1995.

6. A.D. Oliver, W.G. Baer, and K.D. Wise, "A Bulk-Micromachined 1024-Element Uncooled Infrared Imager", Proc. of the 8th Int. Conference on Solid-State Sensors and Actuators (Transducers '95), and Eurosensors IX, pp. 636-639, 1995.

7 M. L. Wilson, D. Kubisiak, R. A. Wood, J. A. Ridley and M. Listvan, "An Uncooled Thermo-Electric Microthermopile Camera Developed Using Silicon Microstructure Detectors", Proc. IRIS Specialty Group on Infrared Detectors, vol. II,
pp. 215-225, August 1991.

8. R.A. Wood, T.M. Rezachek, P.W. Kruse and R.N. Schmidt, "IR SnapShot ${ }^{\mathrm{TM}}$ Camera", Proc. SPIE 2552, Infrared Technology XXI, pp. 654-660, 1995.

9. M.C. Foote, E.W. Jones, and T. Caillat, "Uncooled Thermopile Infrared Detector Linear Arrays with Detectivity Greater Than $10^{9} \mathrm{cmHz} 1 / 2 / \mathrm{W}^{\prime}$, IEEE Transactions on Electron Devices, vol. 45, no. 9, pp. 1896-1902, September, 1998.

10. M.C. Foote and E.W. Jones, "High Performance Micromachined Thermopile Linear Arrays", Proc. SPIE 3379, Infrared Detectors and Focal Plane Arrays V, pp. 192-197, 1998. 